\title{
Uncommon Pulmonary Metastases and Metastasectomy: A Review
}

\author{
Kanthan $\mathrm{R}^{1 *}$, Senger $\mathrm{JL}^{1}$ and Kanthan $\mathrm{SC}^{2}$
}

${ }^{1}$ Departments of Pathology and Laboratory Medicine, Royal University Hospital, University of Saskatchewan, Saskatoon, Saskatchewan, Canada ${ }^{2}$ Departments of Surgery, Royal University Hospital, University of Saskatchewan, Saskatoon, Saskatchewan, Canada

\begin{abstract}
Background: The development of pulmonary metastases is a critical step in oncology management as it is a major determinant of survival for patients with cancer. Primaries that commonly metastasize to the lung arise from breast, head/neck, and the gastrointestinal tract. The aim of this study was to review the pathobiogenesis of pulmonary metastases and the evolution of curative pulmonary metastasectomy as reported in the literature in addition to analyzing in detail uncommon pulmonary metastatic lesions received over a period of 14 years.
\end{abstract}

Design: The indexed uncommon pulmonary metastases were reviewed and analyzed in detail. A 14-year (1996-2010) computer-based review using the Laboratory-Information-System was conducted in our laboratory. Results were categorized based on age, sex, and primary sites of origin with special emphasis to study unusual pulmonary metastases.

Results: 230 cases of pulmonary metastases were retrieved on review. 129 females (56\%) and 101 males (44\%) ranging in age from 19 months to 91 years (median 65 years) were identified with their primary sites of origin being: breast $(28.3 \%)$, gastrointestinal tract $(27.8 \%)$, kidney $(8.7 \%)$, and melanoma $(7.0 \%)$. Three uncommon diagnoses were identified and studied in detail: 1) an index case of metastatic benign pleomorphic adenoma, case series of 2) endometrial stromal sarcoma and 3) osteosarcoma.

Conclusion: Due to the poor prognosis of metastatic lung cancer, early recognition with accurate diagnosis is an important step for optimal patient management. In this context, pathologic awareness of uncommon metastases remains a challenging task. Pulmonary metastasectomy is a curative option for an increasing number of patients due to recent advances in chemotherapy that achieve locoregional control of the primary tumours. With this increased number of pulmonary metastasectomy, recognition of not only 'common' pulmonary malignancies but also more rare entities becomes central to best practices in surgical pathology.

\section{Background}

Though the leading cause of cancer death among both sexes in Canada is lung cancer [1], the most common neoplasm in the lung is pulmonary metastases. Autopsy reports indicate $20-30 \%$ of all patients with neoplastic diseases of a malignant nature will demonstrate such lesions [2]. Pulmonary metastases occur when tumour cells detach from the primary and grow into the lungs by attaching to the basement membrane [3]. Once established in the parenchyma, local or extensive growth of the metastases results in the formation of a tumour [4]. Lung metastasis only develops from a small proportion of tumour cells arriving in the lungs by blood [5]. Most patients with metastasis to the lung are asymptomatic [4], and symptoms that do arise do not immediately appear; therefore upon clinical recognition, it is often too late for curative surgical resection [2]. Pulmonary metastasis is a diagnosis with poor prognosis, as it usually indicates extra-thoracic involvement of a primary tumour elsewhere. Ineffective systemic therapy also contributes to lowered survival rate. Such metastatic pulmonary lesions may be treated with chemotherapy, radiation therapy, immunotherapy, and surgery, with resection acting as the principle method of aggressive treatment with a curative goal [2].

First performed by Weinlechner [6] almost 125 years ago, pulmonary metastasectomy has been slow to gain acceptance [7]. Since 1882, imaging techniques for surveillance have improved dramatically, leading to earlier detection of these silent lesions and thus facilitating surgical treatment. With combination chemotherapy, pulmonary metastasectomy has become a part of a multi-factional treatment regime that offers the greatest hope for a cure [7]. As the literature validates the effectiveness of this treatment option, it is likely that as rates of pulmonary metastasectomies continue to rise there will be an increased number of surgical pathology lung specimens in the laboratory. In this context, awareness of the clinical and pathologic features of common and uncommon metastatic lesions is important to optimize future patient care.

The aim of this study was to review of the pathobiogenesis of pulmonary metastases and the evolution of curative pulmonary metastasectomy as reported in the literature in addition to analyzing in detail uncommon pulmonary metastatic lesions received over a period of 14 years.

\section{Methods}

A comprehensive search of the Laboratory-Information-System (LIS) was used to identify patients with metastatic pulmonary lesions, with the exclusion of all hematopoietic malignancies. All surgical pathology reports were reviewed, analyzed, and categorized based on the histological-type and site of the primary neoplasm. These categories were then assessed and divided into common and

*Corresponding author: Dr. Rani Kanthan, Room 2868, G-Wing, Royal University Hospital, 103 Hospital Drive, Saskatoon, SK, Canada, S7N 0W8, Tel: 306-6332158; Fax: 306-655-2223; E-mail: rani.kanthan@saskatoonhealthregion.ca

Received July 11, 2011; Accepted October 24, 2011; Published October 26 2011

Citation: Kanthan R, Senger JL, Kanthan SC (2011) Uncommon Pulmonary Metastases and Metastasectomy: A Review. J Cancer Sci Ther S11. doi:10.4172/1948-5956.S11-001

Copyright: ( $) 2011$ Kanthan R, et al. This is an open-access article distributed under the terms of the Creative Commons Attribution License, which permits unrestricted use, distribution, and reproduction in any medium, provided the original author and source are credited. 
Citation: Kanthan R, Senger JL, Kanthan SC (2011) Uncommon Pulmonary Metastases and Metastasectomy: A Review. J Cancer Sci Ther S11. doi:10.4172/1948-5956.S11-001

uncommon pulmonary lesions in congruent with the literature. The incidence of each type of lesion within both categories was calculated. The histopathological slides were retrieved and reviewed. Uncommon pulmonary metastases were analyzed carefully, and index cases of metastasizing benign pleomorphic adenoma, endometrial stromal sarcoma and osteosarcoma were studied in detail.

A literature search was conducted using the National Library of Medicine interface PubMed. The search terms "pleomorphic adenoma" or "metastasizing mixed" coupled with "lung" or "pulmonary" were selected. PubMed's 'Related Articles' feature was used to extend our search. Articles obtained from their bibliographies and secondary references were identified and retrieved. The same process was carried out to identify literature regarding endometrial stromal sarcoma, using the key words "endometrial stromal sarcoma" and "lung metastas". The evolution of the role of pulmonary metastasectomy as a method for curative treatment was reviewed with special focus on uncommon pulmonary lesions.

This study was conducted with ethics approval from the University of Saskatchewan Biomedical Research Ethics Review Committee.

\section{Results}

\section{4-year surgical review}

A review of fourteen years (1996-2010) revealed a total of 230 cases of pulmonary metastases; of these 177 (80\%) were histopathology surgical specimens and 53 (20\%) were fine needle aspiration cytology. Patient's age ranged from 19 months to 92 years, averaging 61 years (median 65 years). 129 (56\%) patients were female, and 101 (44\%) were male. In our series, breast (65 cases, $28.3 \%$ ) and gastrointestinal tract (64 cases, 27.8\%) were the most common primaries, followed by the kidney (20 cases, $8.7 \%$ ) and melanoma (16 cases, $7.0 \%)$. Other primary organ sites included thyroid (10 cases, $4.3 \%)$, uterus [7 cases total (3.0\%)], testicle [ 5 cases total (2.2\%)], bone ( 4 cases, $1.7 \%$ ), prostate ( 4 cases, $1.7 \%)$, ovary ( 2 cases, $0.9 \%)$, squamous cell carcinoma ( 2 cases, $0.9 \%$ ), Ewing's sarcoma ( 2 cases, $0.9 \%$ ) and pancreas (3 cases, $1.7 \%$ ). 4 cases $(1.7 \%)$ were determined to be undifferentiated high-grade malignancies originating from either the breast, colon, ovary, thyroid, kidney, or prostate. Uncommon primaries seen in our series included: neuroblastoma (1), malignant peripheral nerve sheath tumour (2), cholangiocarcinoma of the bile duct (1), squamous cell carcinoma of the cervix (1), chordoma (1) and metastasizing benign pleomorphic adenoma (1). The pathological material in all cases was reviewed. As the unusual metastases from chordoma and squamous cell carcinoma of the cervix have been previously reported from our laboratory $[8,9]$ we elected to further elucidate the findings of metastatic benign pleomorphic adenoma (1), endometrial stromal sarcoma (4), and osteosarcoma (4).

\section{Uncommon pulmonary metastases}

Metastatic benign pleomorphic adenoma (1 case): A 58 yearold female with a nine-year history of chronic metastasizing benign pleomorphic adenoma, presented initially with a submandibular mass that reoccurred twice locally before metastasizing to the T-12 spine where it caused paraplegia. Repeated spinal recurrences were treated by surgical decompression. Postoperative CT scans of the thorax revealed a large amorphous soft tissue mass extending into the posterior mediastinum bilaterally with involvement of the lung parenchyma which was confirmed with a biopsy to be metastatic in origin. The patient died within a few months.
Histopathological analysis of the original submandibular specimen, spinal lesion and the lung mass showed identical features of a biphasic tumour composed of the epithelial component with ductal structures admixed with the mesenchymal-like component of mucoid/ myxoid/cartilaginous stroma (Figure 1a,b,c). There was no evidence of increased mitotic activity or progressive cellular or nuclear atypia in the lung sections (Figure 1d).

Endometrial Stromal Sarcoma (4 cases): Four cases of endometrial stromal sarcoma (ESS) were identified in our review. Patients were aged 49, 50, 66 and 69 and were equally distributed in the right and left lungs. In two cases the lung lesion was composed of wellcircumscribed, capsulated nodules (Figure $2 \mathrm{a}$ ) and the other two were ill-defined non-encapsulated masses (Figure 2b).

Histopathological analysis of all cases revealed identical findings of the presence of a spindle cell lesion arranged in ill-defined whorls, composed of fairly uniform cells with mild nuclear atypia and a growth pattern highly reminiscent of endometrial stromal cells (Figure 2c,2d). Immunohistochemically these neoplastic cells showed strong staining to estrogen and progesterone receptor antibodies with diffuse cytoplasmic staining for CD10 and vimentin. In three of the four cases the lung lesions closely resembled the original primary uterine lesion.

Osteosarcoma (4 cases): Four cases of osteosarcoma were identified, three of which were paediatric in origin. Patients were aged $10(\mathrm{~F}), 10(\mathrm{~F}), 16(\mathrm{M})$, and $42(\mathrm{M})$ years. Specimens received in the laboratory included wedge resection of the left lung (1), multiple nodules in the right lung (1), and partial resection of right and left lobes of the lungs (2).

Histopathological examination of all cases confirmed the presence of malignant cells forming osteoid establishing the diagnosis of osteogenic sarcoma (Figure 3a). The matrix varied between osteoblastic in one case as seen in Figure $3 \mathrm{~b}$ and chondroblastic in another as seen in Figure $3 \mathrm{c}$. The lesional cells were a heterogeneous mixture of spindle,
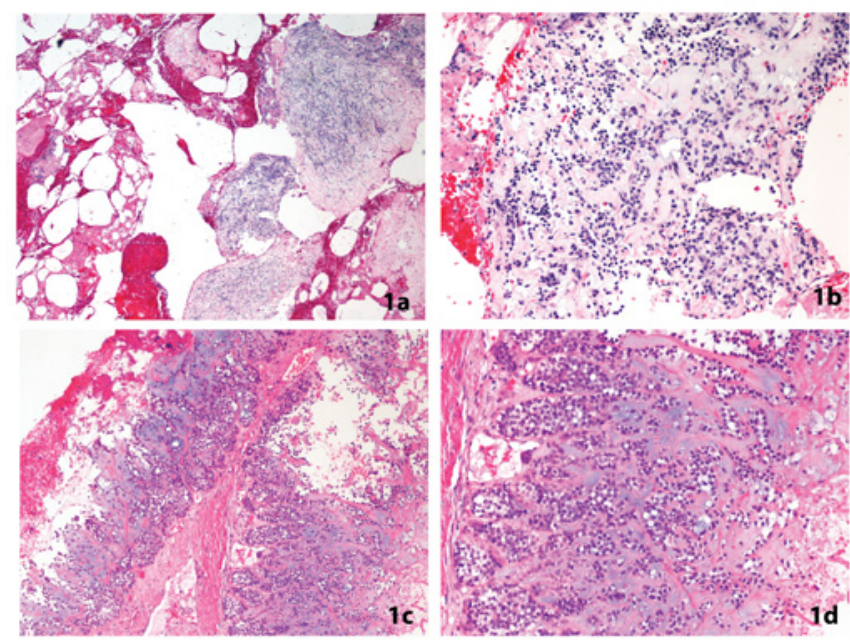

Figure 1a,b,c,d: Metastasizing benign pleomorphic adenoma in the lung. Photomicrographs of hematoxylin and eosin stained slides at low magnification (objective lens $\times 4$ ) shows nodules of benign pleomorphic adenoma (1a) composed of epithelial elements in a rich chondromyxoid background $(1 \mathrm{~b} ; 1 \mathrm{c})$ with no evidence of nuclear or cellular atypia (1d). 

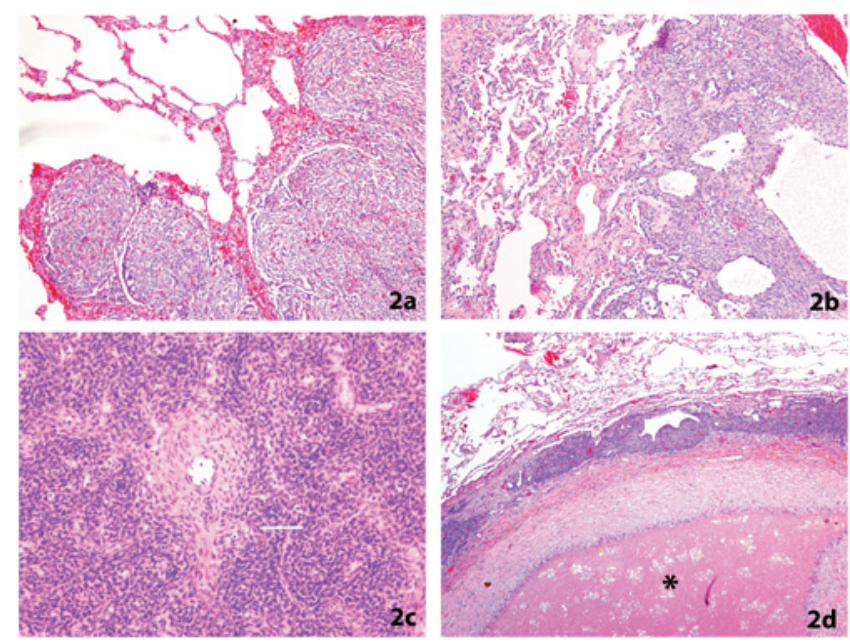

Figure 2a,b,c,d: Endometrial stromal sarcoma in the lung. Photomicrographs of hematoxylin and eosin stained slides at low magnification (objective lens $\mathrm{x} 4$ ) shows well-circumscribed capsulated (2a) and ill-defined non-encapsulated (2b) tumour nodules in the lung. Medium magnification (objective lens $\times 10$ ) shows the whorling pattern of the lesional cells $(2 \mathrm{c})$ with large areas of necrosis $\left(^{*}\right)$ in Figure $2 \mathrm{~d}$.
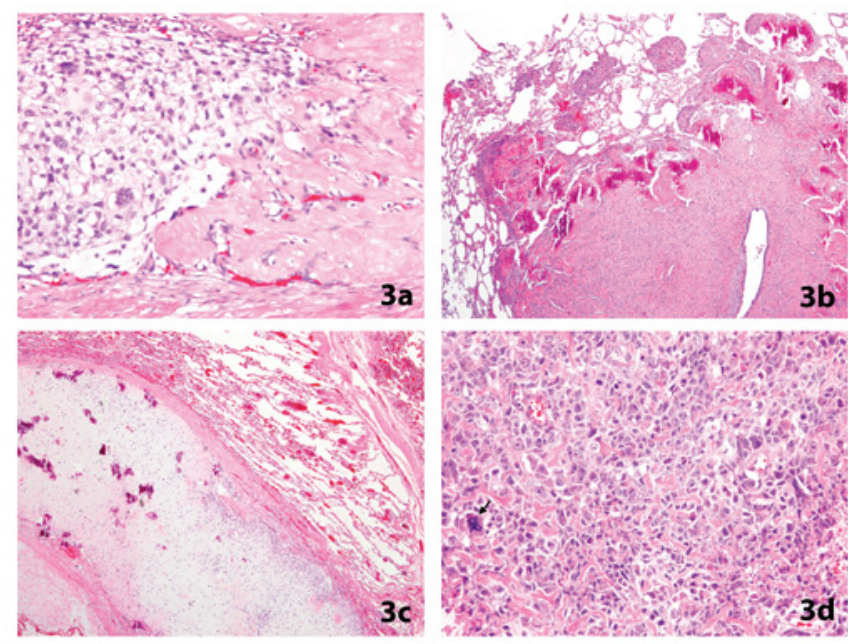

Figure 3a,b,c,d: Osteosarcoma in the lung. Photomicrographs of hematoxylin and eosin stained slides at medium magnification (objective lens $\mathrm{x} 10$ ) shows malignant cells forming osteoid (3a) with an osteoblastic matrix in Figure $3 \mathrm{~b}$ and a chondroblastic matrix in Figure 3c. High magnification (objective lens $\times 20$ ) in Figure $3 d$ shows the presence of malignant hyperchromatic cells with marked cellular and nuclear atypia with abnormal mitosis $(\boldsymbol{\nabla})$.

fusiform, epithelioid, and multinucleated giant cells with classical malignant features of hyperchromatic cellular and nuclear atypia, with increased abnormal mitosis and necrosis (Figure 3d).

\section{Discussion}

The lungs are the organ most frequently affected by metastatic disease [10], and secondary tumours occur in $20-50 \%$ of patients with a non-pulmonary primary [11]. Five features of the pulmonary system have been identified that may contribute to the heightened incidence of such metastases. These include: a) it continually retrieves the entire right-sided cardiac output, b) it contains a high-density capillary bed, c) it is the first capillary plexus met by cells after lymphatic drainage enters the venous system, and d) it consist of delicate membranes that may draw on oxygenated air to promote sustenance $[12,13]$. The high levels of vascularization and richness of oxygen in the lungs provides an environment rich in the nutrients required for neoplastic growth [14]. Autopsy series demonstrate that $20-54 \%$ of patients who died from malignant disease had pulmonary metastases; however, the reported clinical consequence and the impact on quality of life remain variable [15]. Most patients with pulmonary metastases are asymptomatic, with only $5-15 \%$ developing symptoms over time such as dyspnoea, cough, hemoptysis, chest pain, or a pneumothorax [11]. Hemoptysis suggests endobronchial metastases, while dyspnoea is believed to be related to the tumour replacing the parenchyma, and pain may signify involvement of the parietal pleura [11]. Additionally, pneumothoraces present in up to $10 \%$ of patients, particularly those with a sarcomatous primary [11].

Common extrathoracic primary origins include the kidney (92\%), skin $(78 \%)$, breast $(72 \%)$, thyroid $(57 \%)$, pancreas $(56 \%)$, prostate (50\%), stomach (47\%), uterus (44\%), colon (41\%) [16]; however, virtually any primary malignancy can metastasize to the lung [12] In our surgical pathology review over the past 14 years, the common sites of the primary lesion included the breast (28.3\%), gastrointestinal system (27.8\%), kidney (8.7\%), skin (7.0\%), and thyroid (4.3\%). These incidences are less frequent than those reported in the literature. Our series did not confirm any lesions consistent with origin from the stomach. Such discrepancies may be related to a small sample size.

Adenocarcinomas have a greater propensity for this pattern of dispersion than other extra-thoracic solid tumours, as seen in our surgical review [12]. Metastases to the lung may take many forms: solitary and multiple parenchymal nodules, lymphangitic carcinomatosis [17], tumour emboli, endobronchial metastases, and pleural effusions [18]. Dissemination of these primaries chiefly occurs via haematogenous spread [10]. Malignant cells may enter the venous blood either directly or through the lymph drainage, and this pathway is generally attributed to metastases from the head/neck, thyroid, adrenal, kidney, testes, melanoma, or osteosarcoma [13]. Less common forms of dissemination include seeding through the metastatic involvement of other organs and direct extension of the cancer through lymphatics that traverse those structures $[13,17]$.

Metastatic lung cancer is associated with a poor prognosis, and best-practice management involves a multidisciplinary approach with the oncologist, a thoracic surgeon, and a subspecialty radiologist. Typical features of lung metastases depend heavily on the origin and type of primary, and often includes cavitation, calcification, and either a diffuse miliary seeding or a large single metastasis [10]. These lesions are commonly detected on chest $\mathrm{x}$-ray (CXR) as solitary or multiple well-circumscribed, pulmonary nodules with a broad range of size and shape [15]. Differential benign diagnoses include granulomas, sterilised metastases, infection, and proliferation of intrapulmonary nodes $[10,13]$. Modern imaging equipment has enhanced our ability to understand what is occurring within an internal organ, and as such it should come as no surprise that thin collimated helical scans are able to detect $20-25 \%$ more pulmonary lesions than the ordinary CT scan [7]. In addition, a PET scan with F-labeled fluoro-2-deoxy-D-glucose is able to detect neoplastic disease with a pulmonary sensitivity of more than $90 \%$ for nodules greater than $7 \mathrm{~mm}$ [7]. Increasing specificity and sensitivity in these methods of detection may provide an early 
Citation: Kanthan R, Senger JL, Kanthan SC (2011) Uncommon Pulmonary Metastases and Metastasectomy: A Review. J Cancer Sci Ther S11. doi:10.4172/1948-5956.S11-001

Page 4 of 7

\begin{tabular}{|c|c|c|c|}
\hline Histological Diagnosis & Site of Primary & Frequency & Percentage \\
\hline Adenocarcinoma & Breast & 65 & $28.3 \%$ \\
\hline Adenocarcinoma & Gastrointestinal (colorectal) & 64 & $27.8 \%$ \\
\hline Adenocarcinoma & Prostate & 4 & $1.7 \%$ \\
\hline Adenocarcinoma & Pancreas & 3 & $1.3 \%$ \\
\hline Cholangiocarcinoma & Bile ducts & 1 & $0.4 \%$ \\
\hline Serous adenocarcinoma & Ovary & 2 & $0.9 \%$ \\
\hline Urothelial carcinoma & Kidney & 20 & $8.7 \%$ \\
\hline Papillary carcinoma & Thyroid & 10 & $4.3 \%$ \\
\hline Melanoma & Skin & 16 & $7.0 \%$ \\
\hline Squamous cell carcinoma & Skin & 1 & $0.4 \%$ \\
\hline Squamous cell carcinoma & Base of tongue & 1 & $0.4 \%$ \\
\hline Squamous cell carcinoma & Cervix & 2 & $0.9 \%$ \\
\hline Squamous cell carcinoma & Esophagus & 1 & $0.4 \%$ \\
\hline Endometrioid carcinoma & Endometrium & 3 & $1.3 \%$ \\
\hline Endometrial stromal sarcoma & Endometrium & 4 & $1.7 \%$ \\
\hline Leiomyosarcoma & Uterus & 3 & $1.3 \%$ \\
\hline Leiomyosarcoma & Inferior vena cava & 1 & $0.4 \%$ \\
\hline Leiomyosarcoma & Left leg & 2 & $0.9 \%$ \\
\hline Seminoma & Testicle & 2 & $0.9 \%$ \\
\hline Embryonal Carcinoma & Testicle & 2 & $0.9 \%$ \\
\hline Rhabdomyosarcoma & Testicle & 1 & $0.4 \%$ \\
\hline Adenoid Cystic Carcinoma & Salivary gland- parotid & 1 & $0.4 \%$ \\
\hline Metastasizing pleomorphic adenoma & Salivary gland-submandibular & 1 & $0.4 \%$ \\
\hline Osteosarcoma & Femur & 4 & $1.7 \%$ \\
\hline Chondrosarcoma & Right Femur & 2 & $0.9 \%$ \\
\hline Ewing's sarcoma & Chest wall mass & 2 & $0.9 \%$ \\
\hline Hepatocellular carcinoma & Liver & 2 & $0.9 \%$ \\
\hline Neuroblastoma & Adrenal & 1 & $0.4 \%$ \\
\hline Malignant peripheral nerve sheath tumour & Right neck & 2 & $0.9 \%$ \\
\hline Malignant Mesothelioma & Mesothelium & 1 & $0.4 \%$ \\
\hline Liposarcoma & Thigh & 1 & $0.4 \%$ \\
\hline Chordoma & Spine & 1 & $0.4 \%$ \\
\hline $\begin{array}{l}\text { Undifferentiated high-grade malignancy- poorly differentiated } \\
\text { adenocarcinoma }\end{array}$ & $\begin{array}{l}\text { Breast, colon, ovary, thyroid, kidney, } \\
\text { prostate or GI }\end{array}$ & 4 & $1.7 \%$ \\
\hline \multicolumn{2}{|l|}{ TOTAL } & 230 & $100 \%$ \\
\hline
\end{tabular}

Table 1: A 14 Year Surgical Pathology Review of Pulmonary Metastases (Saskatoon Lab Information System 1997-2010).

indication of pulmonary spread, thus offering an opportunity for curative pulmonary metastasectomy whereas in the past such lesions were more commonly detected only at autopsy.

The presence of a single pulmonary nodule presents diagnostic difficulty, as incidence of solitary primary lung lesions is more common than solitary metastatic lung lesions [10]. One-fifth of all pulmonary metastases will present as solitary nodules with no evidence of involvement of either the lymphatics or extra-pulmonary organs [11]. Metastatic lesions are generally a) smaller than primaries b) grow more rapidly, c) located more in the lower lobes and outer parts of the lungs and d) have more well-circumscribed margins than primary malignant tumours [16]. Over $80 \%$ of metastatic lesions are less than 1 $\mathrm{cm}$, which is the lower limit of CXR detection [11]. As earlier detection of pulmonary metastases continues to increase due to technological advancements and pulmonary metastasectomy gains acceptance, the number of lung specimens arriving in the surgical laboratory is correspondingly increasing. When metastatic disease is suspected in a pulmonary lesion, comparison with the primary lesion supplemented by ancillary techniques such as immunohistochemical studies may help in confirming the exact diagnosis. Such accurate pathological diagnosis is clinically essential to guide treatment protocols.

Four possible treatment plans for pulmonary metastases include chemotherapy, immunotherapy, radiotherapy, and surgical resection. Chemotherapy, though rarely curative, may postoperatively aid in the 
control of systemic micro-metastases. When combined with resection, it improves the prognosis for patients with sarcomas, testicular, colorectal, and renal carcinoma. In addition, its neo-adjuvant use may reduce tumour burden improving surgical resectability [7]. Immunotherapy is the selected treatment for certain metastatic lesions that express immunogenic antigens as in melanoma, in which it may serve as front-line therapy [2]. Radiotherapy is not normally associated with an increase in survival as the required dose for tumour control is greater than the tolerance level of the pulmonary tissue; therefore, this treatment is purely palliative [2]. Though their success rates do not yet match those of surgical intervention, stereotactic body radiation therapy and radiofrequency ablation are options for patients at a high surgical risk with reported 1-year survival rates of $85 \%$ [14]

The first surgical removal of a pulmonary metastasis was carried out in 1822 by Weinlechner [6] and since then the goal of this procedure has been to eradicate tumour with preservation of the lung parenchyma to the greatest possible extent. A complete resection requires all surgical margins to be microscopically negative, and is a strong predictor of improved survival [7]. It was initially believed that pulmonary metastases were indicative of systemic dissemination, with surgical resection providing no survival benefit. It is now believed that under the proper conditions, complete pulmonary metastasectomy offers a potentially curative treatment [2,19]. An expansion of Alexander and Haight's [20] criteria that must be fulfilled for a patient to be eligible for curative pulmonary metastasectomy are: a) no disease present at the primary tumour site, b) no metastases present outside the lungs, c) no non-resectable pulmonary nodules present, d) no nonsurgical alternative for cure, and e) the patient should be able to tolerate the surgical treatment [15]. Achieving the first criteria is highly dependent on the histological nature of the primary, as sarcomas, renal cell cancers and head and neck cancers will often spread preferentially to the lungs whereas melanoma, breast and colon cancers are more widely disseminated [14]. Pulmonary metastasectomy may be carried out beginning four weeks after complete resection of the primary lesion, and patients with bilateral metastases should first undergo resection of the least-involved lung to determine the resectability of the lesion [2]. 'Resectability' encompasses the location, status, and number of lesions, as well as individual patient factors [7]. Most pulmonary metastases are either locally unresectable or synchronous with other metastases with only $1 \%$ of pulmonary tumours being resectable [4]. Survival is optimized by this treatment when tumours are solitary and less than $3 \mathrm{~cm}$ in diameter [15]. 3-year success rates as high as $78 \%$, and 10 -year survival rates of $26 \%$ have been reported [14]. Patients with a sarcomatous primary lesion generally benefit the most from pulmonary metastasectomy due to the tumour's high propensity for exclusive pulmonary spread [7].

Video-assisted thoracoscopic surgery (VATS) remains a controversial approach to the treatment of pulmonary metastases. This newest approach confers excellent surgical access, reduced hospital stay, minimal postoperative pain, and improved long-term quality of life. These results may be attributed to a reduced incision length $(5-8 \mathrm{~cm})$ compared with that of tumour resection $(30-40 \mathrm{~cm})$ which correlates with a reduction of systemic stress [7]. The downside includes the possibility of small nodules going undetected as the procedure does not allow lung palpation, and consequently tumours may recur along the thoracoscopic surgical tracts $[14,15]$. Open thoracotomy has been shown to be more capable of detecting pulmonary lesions when compared to VATS [5].
Prognostic indicators including site and type of primary, length of disease-free interval, number of metastases identified, and doubling time of the tumour are criteria used to select patients for metastasectomy $[2,4]$. To be considered, the primary tumour must be under control with no evidence of widespread extra-pulmonary metastases, and the patients must be expected to have sufficient pulmonary function postoperatively [4]. When metastases involve the pleura, chest wall, or other intra-thoracic structures, en-bloc resection or pneumonectomy may be required [4]. Completeness of resection is the primary factor in determining the rate of local recurrence and long-term survival [5]. Up to $30 \%$ of patients with pulmonary metastases are reported with positive mediastinal lymph nodes, a finding that lowers the 3-year survival rates by $30 \%$ [5].

We will now discuss the index cases selected for detail review in this study.

Metastatic benign pleomorphic adenoma (1 case): Mixed tumours, or pleomorphic adenoma (PA) is the commonest benign neoplasm, representing $3.6 \%$ of all salivary gland tumours. Malignancy of PA can be classified into three categories: a) carcinoma ex pleomorphic adenoma (CXPA): carcinoma arising in the background of PA b) carcinosarcoma: biphasic malignant tumour of the epithelial and mesenchymal components and c) metastasizing malignant pleomorphic adenoma (MPA) [21]. Also known as metastasizing benign pleomorphic adenoma, the paradoxical nature of MPA poses the evident question of how a benign lesion can metastasize. The neoplasm is composed of two components: benign ductal structures and myoepithelial cells from an epithelial element and a metaplastic mesenchymal element with fibrous/chondroid/myxoid features [22]. Such distant metastases are often histologically identical to the primary PA [23,24]. Prior to metastases, $90 \%$ of MPA will have reoccurred several times at the primary site, with the reported length of time interval between the occurrence of the primary and the metastatic lesions averaging 16 years $[21,25,26]$. MPA has been associated with metastases to the bone, lung, lymph nodes, kidney, liver, central nervous system, retroperitoneum, and skin, and spreads haematogenously rather than lymphatically [25]. Reports indicate a relationship between incomplete surgical excision of the primary tumour and the incidence of distant 'metastases'. Further, as chemotherapy and radiotherapy are generally ineffective in combating this disease, surgery is the treatment of choice, and is associated with a survival advantage [25-27]. A relevant clinical history is essential for the accurate pathological diagnosis of such rare pulmonary lesions [25].

Endometrial stromal sarcoma (4 cases): Endometrial stromal sarcoma (ESS) is a rare tumour representing $20 \%$ of uterine sarcomas [28] and only $0.2 \%$ of uterine malignancies [29,30]. Depending on the highest mitotic count, ESS is divided into two categories: low-grade ( $<10$ mitoses/high power field) accounting for $2 / 3$ of ESS diagnosis or high-grade ( $>10$ mitoses/high power field) comprising the other $1 / 3$ $[29,31]$. Low-grade ESS is more common and slow-growing with a 5 -year survival of $80-100 \%$ whereas the high-grade has a more aggressive course with a $37-60 \%$ recurrence rate [32]. On average, women are $40-$ 45 years old, and most present at clinical stage I [31]. ESS recurrence develops in a third to half of patients, and the time interval after the initial treatment may be as long as 30 years [29]. Recurrence is often limited to the pelvis, and abdomen [31]. The incidence of extra-pelvic spread with pulmonary metastases varies from $7-28 \%$, commonly presenting as multiple pulmonary nodules [33]. Metastatic pulmonary ESS may be an incidental finding in an asymptomatic person, or a 
Citation: Kanthan R, Senger JL, Kanthan SC (2011) Uncommon Pulmonary Metastases and Metastasectomy: A Review. J Cancer Sci Ther S11. doi:10.4172/1948-5956.S11-001

Page 6 of 7

patient may present with chest pain, dyspnea, pleural effusion, and/ or pneumothorax [28]. The development of pulmonary metastases is believed to have little effect on patient survival [34]. In the lung, ESS is normally seen as well-circumscribed multiple nodules within air spaces lined by non-neoplastic epithelium; however, abnormal patterns of growth reported include solitary nodules and lymphangitic bilateral spontaneous pneumothroraces [33]. Diagnosis may be complicated by a metastatic lesion that morphologically does not resemble the original uterine ESS [28]. Surgical management of metastatic ESS should be followed by progestin therapy as this tumour is hormonally sensitive [29]. Nodal involvement is reported in $33-45 \%$ of patients; therefore, a role for lymphadenectomy has also been proposed [32].

Osteosarcoma (4 cases): The recent death of Betty Fox (June 17, 2011), mother of national hero Terry Fox, has again reminded Canadians of the devastating outcome of metastatic osteosarcoma. In 1977 at the age of 18 Fox was diagnosed with osteosarcoma and his right leg was amputated. He was frustrated with the paucity of funds dedicated to cancer research, so planned to run across Canada to raise cancer awareness. He did not complete his run because the cancer metastasized to his lungs, and he died soon afterwards in 1981.

Osteosarcoma is the most common primary bone malignancy, usually occurring in the distal femur and proximal tibia and less commonly in the proximal humerus, middle and proximal femur, and non-extremity sites [35].

At initial diagnosis 11-20\% of patients have pulmonary metastases and up to $50 \%$ will develop them later, accounting for $85 \%$ of patients with metastatic osteosarcoma [36,37]. Long-term survival rates for patients with osteosarcoma have been steadily increasing due to the development of intensive regimens of multi-agent chemotherapy, aggressive surgical techniques and innovative diagnostic equipment [36].

With the advent of neo-adjuvant chemotherapy, modern trends in the management of osteosarcoma include limb-salvage surgery and pulmonary metastasectomy of chest nodules. Such changes in treatment regime now provide an increased number of pulmonary pathological specimens with malignant bone-forming lesions which have to be recognized as being consistent with metastatic osteosarcoma. Overall survival rates decrease substantially from $60-70 \%$ if the osteosarcoma is localized to $10-30 \%$ if metastatic [35]. Since the introduction of multimodality therapy, 5-year survival rates have increased from 10 $20 \%$ to $32-40 \%$ [38]. Despite this success, the majority of these cancers have a high incidence of recurrence within one year of surgical resection and up to $85 \%$ will eventually relapse with pulmonary disease [38].

\section{Conclusion}

Pulmonary metastases are a significant cause of cancer-related deaths, and are often detected only at autopsy, especially if they are silent and asymptomatic. However, with recent advances of radiological imaging techniques such as thin collimated helical CT scans and PET scans, detection rates of pulmonary metastases have increased exponentially. This, coupled with strides in chemotherapy and better loco-regional control of the primary tumours, has led to increased practice of 'curative' pulmonary metastasectomy. Due to the poor prognosis of metastatic lung cancer, early recognition with accurate diagnosis is an important step in the therapeutic management of these patients. Additionally, pathological awareness of uncommon metastases such as those from metastasizing benign pleomorphic adenoma, endometrial stromal sarcoma and osteosarcoma though a challenging task is essential for appropriate patient management.

\section{References}

1. Lung Cancer Statistics at a Glance (2011) Canadian Cancer Society.

2. Morton DL, Shen P (2003) Metastatic Tumors in the Thorax. Holland-Frei Cancer Medicine. ( $6^{\text {th }}$ edn), BC Decker, Hamilton.

3. Erhunmwunsee, L, D'Amico T (2009) Surgical management of pulmonary metastases. Ann Thorac Sur 88: 2052-2060.

4. Quiros RM, Scott WJ (2008) Surgical Treatment of Metastatic Disease to the Lung. Semin Oncol 35: 134-46.

5. Demmy TL, Dunn KB (2007) Surgical and nonsurgical therapy for lung metastasis: indications and outcomes. Surgical Oncology Clininics of North America 16: 579-605.

6. Weinlechner JW (1882) Tumoren an der brustwand und deren behnadlung resection der rippeneroffnung der brusthohle und partielle entfernung der lunge. Wein Med Wochenschr 32: 289-291.

7. Sternberg DI, Sonett JR (2007) Surgical Therapy of Lung Metastases. Semin Oncol 34: 186-296

8. Kanthan R, Senger JL, Diudea D (2010) Pulmonary lymphangitic carcinomatosis from squamous cell carcinoma of the cervix. World J Surg Oncol 8: 107.

9. Kanthan R, Senger JL (2010) Fine-needle aspiration cytology with histologica correlation of chordoma metastatic to the lung: a diagnostic dilemma. Diagn Cytopathol [Epub ahead of print].

10. Schueller G, Herold CJ (2003) Lung Metastases. Cancer Imaging 3: 126-128.

11. Meerbeek JP, Gosselin R, Duyck PH (2007) Lung metastases: epidemiology, clinical presentation and imaging. lung metastases and isolated lung perfusion. Nova Science Publishers Inc, New York.

12. Dail DH (2008) Metastases to and from the lung. Dail and Hammar's Pulmonary Pathology, ( $3^{\text {rd }}$ edn) Neoplastic Lung Disease, Springer, New York.

13. Herold CJ, Bankier AA, Fleischmann D (1996) Lung metastases. Eur Radiol 6 596-606.

14. Zheng Y, Fernando HC (2010) Surgical and nonresectional therapies for pulmonary metastasis. Surgical Clinics of North America 90: 1041-1051.

15. Yoneda KY, Louie S, Shelton DK (2000) Approach to pulmonary metastases. Curr Opin Pulm Med 6: 356-363.

16. Nia PS, Colpaert C (2007) Molecular biology and pathology of lung metastases Lung metastases and isolated lung perfusion. Nova Science Publishers Inc, New York.

17. Kanthan R, Senger JL, Diudea D (2010) Pulmonary lymphangitic carcinomatosis from squamous cell carcinoma of the cervix. World J Surg Oncol 8: 107.

18. Avdalovic M, Chan A (2004) Thoracic manifestations of common nonpulmonary malignancies of women. Clin Chest Med 25: 379-390.

19. Kaifi JT, Gusani NJ, Deshaies I, Kimchi ET, Reed MF, et al. (2010) Indications and approach to surgical resection of lung metastases. J Surg Oncol 102: 187 195

20. Alexander J, Haight C (1947) Pulmonary resection for solitary metastatic sarcomas and carcinomas. Surg Gynecol Obstet 85: 129-146.

21. Weissferdt A, Langman G (2009) An intracapsular carcinoma ex pleomorphic adenoma with lung metastases composed exclusively of benign elements: Histological evidence of a continuum between metastasizing pleomorphic adenoma and carcinoma ex pleomorphic adenoma. Pathol Res Pract 206 480-483.

22. Fernandez JR, Micas MM, Tello FJM, Berjon J, Montalvo JJ, et al. (2008) Metastatic benign pleomorphic adenoma. Report of a case and review of the literature. Med Oral Patol Oral Cir Bucal 13: E193-196.

23. Bradley PJ (2001) Distant metastases from salivary glands cancer. ORL $J$ Otorhinolaryngol Relat Spec 63: 233-242. 
Citation: Kanthan R, Senger JL, Kanthan SC (2011) Uncommon Pulmonary Metastases and Metastasectomy: A Review. J Cancer Sci Ther S11. doi:10.4172/1948-5956.S11-001

Page 7 of 7

24. Chen, KTK (1978) Metastasizing pleomorphic adenoma of the salivary gland. Cancer 42: 2407-2411.

25. Raja V, China C, Masaki KH, Nakano G (2002) Benign metastasizing pleomorphic adenoma. J Clin Oncol 20: 2400-2406.

26. Nouraei SA, Ferguson MS, Clarke PM, Sandison A, Sandhu GS, et al. (2006) Metastasizing pleomorphic salivary adenoma. Arch Otolaryngol Head Neck Surg 132: 789-793

27. Manucha V, loffe OB (2008) Metastasizing pleomorphic adenoma of the salivary gland. Arch Pathol Lab Med 132: 1445-1447.

28. Koga T, Ushijima K, Kage M, Ichiki M, Kitajima T, et al. (2006) Pulmonary metastasis of endometrial stromal sarcoma. Kurume Med J 53: 95-97.

29. Garavaglia E, Pella F, Montoli S, Voci C, Taccagni G et al. (2010) Treatment of recurrent or metastatic low-grade endometrial stromal sarcoma: three case reports. Int J Gynecol Cancer 20: 1197-1200.

30. Spano JP, Soria JC, Kambouchner M, Piperno-Neuman S, Morin F, et al. (2003) Long-term survival of patients given hormonal therapy for metastatic endometrial stromal sarcoma. Med Oncol 20: 87-93.

31. Matsuura Y, Yasunaga K, Kuroki H, Inagaki H, Kashimura M (2004) Low-grade endometrial stromal sarcoma recurring with multiple bone and lung metastases: report of a case. Gynecol Oncol 92: 995-998.
32. Gadducci A, Cosio S, Romanini A, Genazzani AR (2008) The management of patients with uterine sarcoma: a debated clinical challenge. Crit Rev Oncol Hematol.65: 129-142.

33. Kim GY, Sung CO, Han J, Park JO, Lee KS (2004) Pulmonary metastases of uterine endometrial stromal sarcoma: diffuse micronodular and ground glass opacities: a case report. J Korean Med Sci 19: 901-903.

34. Aubry MC, Myers JL, Colby TV, Leslie KO, Tazelaar HD (2002) Endometria stromal sarcoma metastatic to the lung: a detailed analysis of 16 patients. Am J Surg Pathol 36: 440-449.

35. Harting MT, Blakely ML (2006) Management of osteosarcoma pulmonary metastases. Semin Pediatr Surg 15: 25-29.

36. Wu PK, Chen WM, Chen CF, Lee OK, Haung CK, et al. (2009) Primary osteogenic sarcoma with pulmonary metastasis: clinical results and prognostic factors in 91 patients. Jpn J Clin Oncol 39: 514-522.

37. Davidson RS, Nwogu CE, Brentjens MJ, Anderson TM (2001) The surgical management of pulmonary metastasis : current concepts. Surg Oncol 10: 35 42.

38. Greelish JP, Friedberg JS (2000) Secondary pulmonary malignancy. Surg Clin North Am 80: 633-657.
This article was originally published in a special issue, Lung Cancer handled by Editor(s). Dr. Harvey I. Pass, NYU Langone Medical Center, Canada 